Annales Geophysicae (2002) 20: 647-653 (c) European Geophysical Society 2002

\title{
Predicted and observed characteristics of small-scale field-aligned irregularities generated in the F-region by low power HF heating
}

\author{
E. Kolesnikova, T. R. Robinson, and J. A. Davies \\ Department of Physics and Astronomy, University of Leicester, Leicester LE1 7RH, UK
}

Received: 20 July 2001 - Revised: 21 December 2001 - Accepted: 8 January 2002

\begin{abstract}
Simultaneous HF scattering from the different regions of the heated volume is used to investigate characteristics of the small-scale field-aligned irregularities in the Fregion. Time of growth, decay rate and saturation level for different pump powers are deduced from the observations and are compared with their behaviour predicted by the thermal parametric instability model. As a result, the estimates of the density and of the temperature modifications inside of the irregularities are obtained.
\end{abstract}

Key words. Ionosphere (ionospheric irregularities)

\section{Introduction}

Experimental studies of the ionosphere, made during the last decades, by means of both ground-based radio sounding techniques and rocket or satellite measurements have revealed that the ionosphere displays strongly irregular structure with the spatial scales in the range from several $\mathrm{cm}$ to hundreds of $\mathrm{km}$. Since 1980, the heating facility near Troms $\varnothing$ has performed systematic heating of the ionosphere with the purpose of investigating the properties of the density irregularities which are excited artificially (see Stubbe, 1996 and references therein). HF waves, which are reflected or scattered from the artificially generated irregularities, provide a powerful diagnostic about scales, density and temporal development of the perturbations and reveal important information about the mechanisms which trigger their growth. Two spatial scales of the density perturbations can be explored using existing radio facilities: (i) large-scale perturbations with a field-perpendicular scale of the order of the heated patch size, and (ii) small-scale field-aligned perturbations with the characteristic transverse dimension of several meters. Largescale perturbations are usually quasi-isotropic and their amplitude and sign strongly depend on the ionospheric altitude (Gurevich, 1978). In the lower ionosphere (below $200 \mathrm{~km}$ ), such density perturbations are significant and are mainly de-

Correspondence to: E. Séran (elena.seran@ @etp.ipsl.fr) termined by the dissociative recombination which leads to the electron density increase due to the heating. In the upper ionosphere (above $300 \mathrm{~km}$ ), the main mechanism, that determines the density perturbations is particle transport across the temperature or density gradients. In such situations, heating is associated with the electron density depletions in the regions of enhanced temperature. At the intermediate heights (between 200 and $300 \mathrm{~km}$ ) the expected amplitudes of the large-scale irregularities are small and might be related to either of the described mechanisms. The dominant process which controls the geometry of small-scale perturbations is electron diffusion along the field line. The ratio between the transverse and field-aligned lengths is of the order of the ratio between the electron collision frequency and gyrofrequency, which is many orders of magnitude less than unity. Therefore, small-scale perturbations are strongly anisotropic and elongated along the magnetic field line. The fact that the small-scale density perturbations grow only during O-mode HF heating supports the idea that the mechanism which is responsible for their growth is the thermal parametric instability (TPI) (Inhester, 1982; Dysthe et al., 1983; Robinson, 1989; Gurevich et al., 1995; Frolov et al., 1997), which is developed at the upper-hybrid frequency range.

In the present paper, we continue a series of investigations devoted to the study of HF-induced striations using the CUTLASS diagnostics (Robinson, 1997, 1998; Bond, 1997) and attempt to find additional arguments in favour of the TPI mechanism. We concentrate our efforts on the investigation of the FAI development under the HF pumping with the low power, which is quite poorly reflected in the literature. In Sect. 2, we review the main results obtained from a simple model of a three-wave interaction, namely the beating between the pump electromagnetic and upper-hybrid electrostatic waves in the presence of the density perturbation. The aim of Sect. 2 is to obtain a rough estimate of the density growth and saturation level as a function of the ratio between the pump electric field and the threshold field. In the Sect. 3, we show how "simultaneous" HF scattering from the different regions of the heated volume may be used to investigate 


\section{SUPERDARN PARAMETER PLOT \\ Hankasalmi: pwr_I}

7 Oct $1997^{280)}$

unknown scan mode $(-6312$

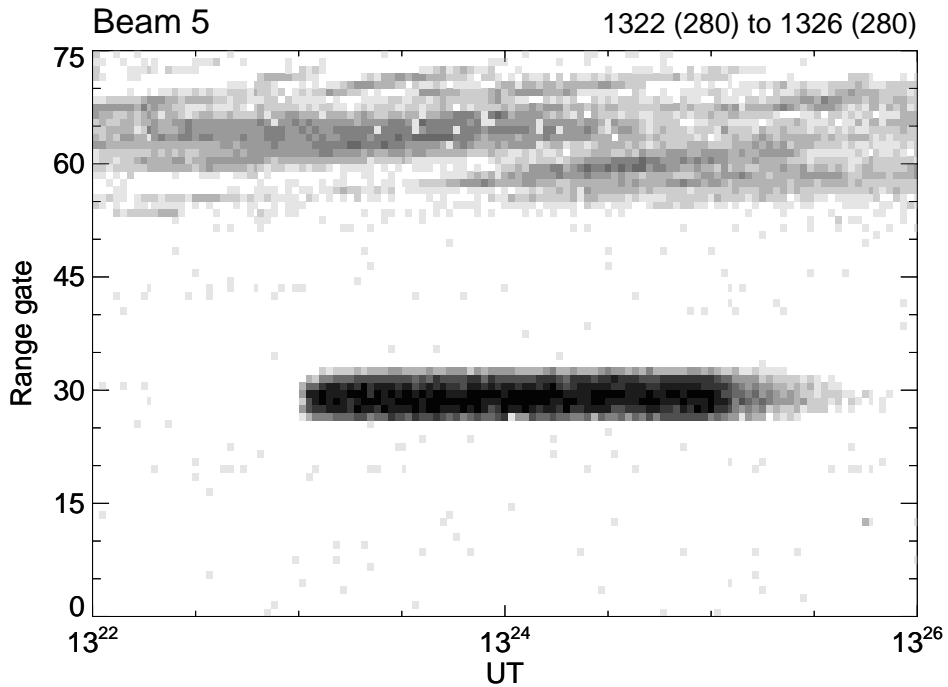

Fig. 1. Power measured in the beam 5 of the HF coherent radar at CUTLASS during $2 \mathrm{~min} \mathrm{HF}$ heating from Troms $\varnothing$ which began at $\sim 13: 23$ UT on 7 October 1997. The power increase is observed in seven gates separated by $\sim 15 \mathrm{~km}$ in the horizontal plane at the altitude of $\sim 230 \mathrm{~km}$. the characteristics of the field-aligned irregularities (FAI); deduce the observed time of growth, decay rate and saturation level for the different pump powers and compare them with the predicted behaviour obtained from the TPI model.

\section{Model of FAI growth and saturation}

Let us assume that modification of plasma density in FAI is caused by the beating between an electromagnetic wave emitted by the heater in O-mode and the electrostatic upperhybrid wave generated by a current perturbation in the heated volume, in which there is a primary small-scale density irregularity $\Delta n_{o}$ with transversal scale $2 \pi k_{\perp}^{-1}$. In this situation, the density variations may be written in the form (see, for example, Robinson, 1989)

$\frac{\partial \Delta n}{\partial t}=D_{2} \Delta n\left(\left(E / E_{t h}\right)^{4} e^{-\sigma \Delta n^{2}}-1\right)$,

where $E$ is the amplitude of the pump electric field at the altitude where the pump frequency matches the upper-hybrid frequency; $E_{t h}$ is the threshold field, $\sigma$ is a function of the pump frequency and the plasma density gradient, $D_{2}=$ $D_{\perp} k_{\perp}^{2}$, in which the transverse thermal diffusion coefficient is defined as $D_{\perp}=T_{e} v_{e} /\left(m_{e} \omega_{c e}^{2}\right)$, where $T_{e}, v_{e}, m_{e}, \omega_{c e}$ are the electron temperature, effective collision frequency, mass and gyrofrequency, respectively. The $e^{-\sigma \Delta n^{2}}$ factor in Eq. (1) arises from the assumption that saturation is caused by the self-anomalous absorption of the pump wave. After integration over time, Eq. (1) reads

$t-t_{o}=\frac{1}{2 D_{2}} \int_{\Delta n_{o}^{2}}^{\Delta n^{2}} \frac{d x}{x\left(\left(E / E_{t h}\right)^{4} e^{-\sigma x}-1\right)}$.
If the pump source is switched off at the moment $t_{o}$, the density perturbation will follow the law

$\Delta n=\Delta n_{o} e^{-D_{2}\left(t-t_{o}\right)}$,

and, therefore, will be damped with a rate which is higher if the transverse size is smaller and the electron temperature is larger.

When the pump source is switched on, an approximate analytical solution may be found in two limits. First, consider the situation when the density perturbation is small, i.e.

$\sigma \Delta n^{2} \ll 1$,

for which the solution of Eq. (2) might be written analytically after development of the exponent, $e^{-\sigma x} \approx 1-\sigma x$. The density perturbation, in this case, becomes

$\Delta n^{2} \approx \frac{a e^{b\left(t-t_{o}\right)}}{c+e^{b\left(t-t_{o}\right)}}$,

in which $a=\sigma^{-1}\left(1-\left(E / E_{t h}\right)^{-4}\right), \quad b=2 D_{2}$ $\left(\left(E / E_{t h}\right)^{4}-1\right), c=a \Delta n_{o}^{-2}-1$. It follows from the solution (5) that the density increases exponentially at the beginning, but after some time thermal diffusion damps its growth.

Second, consider the case when the quantity $\sigma \Delta n^{2}$ is not small, but rather $\sigma \Delta n^{2} \sim 1$, and the pump electric field does not greatly exceed the threshold level at the altitude where the pump frequency matches the upper-hybrid frequency. In this case, the term $\left(\left(E / E_{t h}\right)^{4} e^{-\sigma x}-1\right)$ mainly determines the behaviour of the right side of Eq. (2) and an approximate solution might be deduced by taking the term $1 / x$ out of the integral. Then,

$$
\begin{aligned}
& \Delta n^{2} \approx \sigma^{-1} 1 n\left[\left(E / E_{t h}\right)^{4}\right. \\
& \left.\left\{1-\left[1-\left(E / E_{t h}\right)^{-4} e^{\sigma \Delta n_{*}^{2}}\right] e^{-2 D_{2} \sigma \Delta n_{*}^{2}(t-t *)}\right\}\right] .
\end{aligned}
$$


Here, the symbol " $*$ " is used to mark the quantities that correspond to the moment when $\sigma \Delta n^{2} \sim 1$. After a certain time, which is a function of pump power, the initial perturbation amplitude, the density gradient and thermal diffusion coefficient, the growth of the density perturbation becomes saturated. The dependence of the saturation level on the pump electric field is found to be

$|\Delta n| \approx 2 \sqrt{\sigma^{-1} \ln \left(E / E_{t h}\right)}$.

In the next sections, we will check the estimates deduced from this model of three-wave interaction against the observations.

\section{What we may learn from the HF coherent radar ob- servations}

3.1 Simultaneous scatter from the regions that are heated by electromagnetic waves with different power

Hereafter we assume that the horizontal distribution of the power $(W)$ emitted by the heater into the heated cone (with a half-angle of $\sim 7^{\circ}$ ), which is centered along the direction of the local magnetic field (inclined at $\sim 12^{\circ}$ with respect to the vertical), may be reasonably described by the Gaussian law, i.e.

$W(r) \sim W(0) e^{-r^{2} / a^{2}}$,

where $r$ is the horizontal distance from the center of the heater beam and $a$ is the radius of the heated spot $(\sim 30 \mathrm{~km}$ at the height $230 \mathrm{~km}$ ).

The CUTLASS HF coherent scatter radar located in Finland $\left(62.3^{\circ} \mathrm{N}, 26.6^{\circ} \mathrm{E}\right.$ geographic), $\sim 825 \mathrm{~km}$ from the Troms $\varnothing$ heater $\left(69.2^{\circ} \mathrm{N}, 19.2^{\circ} \mathrm{E}\right.$ geographic), transmits HF waves with a frequency in the range from 8 to $20 \mathrm{MHz}$. The transmitted wave is scattered from the FAI which are generated artificially by heating in the F-region. The received signal is gated into 75 range gates along each beam according to the time delay. Each range gate covers approximately $15 \mathrm{~km}$ in the horizontal plane at the altitude of the wave scattering. The amount of energy scattered from different parts of the heated patch is proportional to the squared density perturbation (Minkoff, 1974) and, therefore, is a function of pump power and according to distribution (8), depends on the distance from the center of the heated region.

Let us consider a typical example of the CUTLASS Finland antenna observations during the $\mathrm{O}$-mode 2 min heating that commenced at $\sim 13: 23$ UT on 7 October 1997 in which the heater was pointed along the magnetic field line and emitted an effective radiated power of $9 \mathrm{MW}$ at the frequency $\sim 4.5 \mathrm{MHz}$. According to the EISCAT incoherent scatter radar measurements, the altitude where the HF pump frequency matches the upper-hybrid frequency is estimated to be $\sim 230 \mathrm{~km}$. At this height, the electron density gradient was $\partial n / \partial h \approx 2 \cdot 10^{6} \mathrm{~m}^{-4}$ and the electron temperature $\sim 2000 \mathrm{~K}$. The CUTLASS radar was operated at the

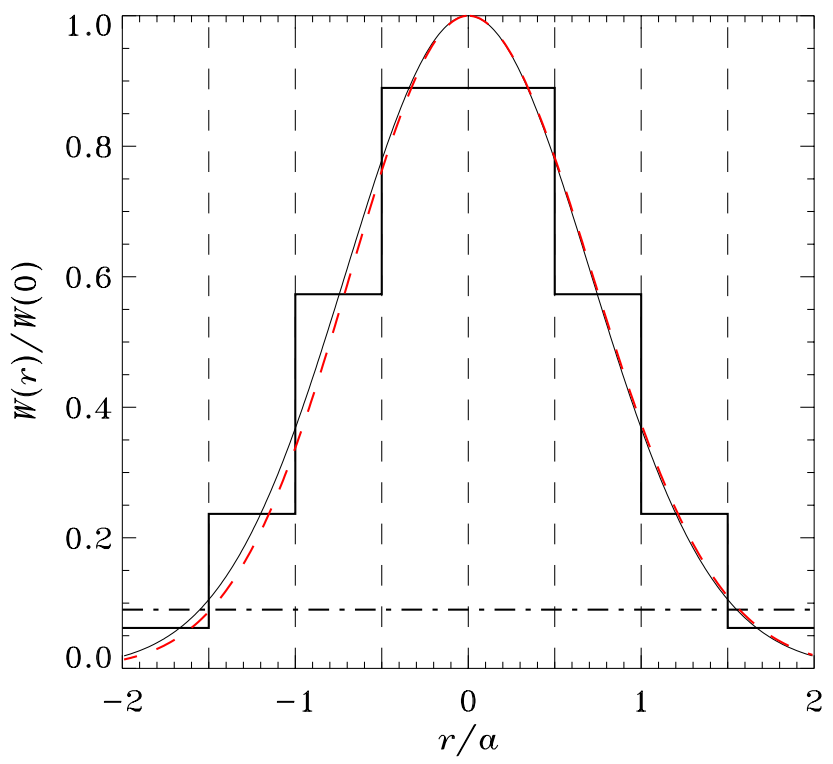

Fig. 2. Distribution of the normalized pump power inside of the heated cone versus the normalized distance from the center for two cases: (i) projection of the heated cone on the horizontal plane at the altitude $230 \mathrm{~km}$ is considered as a perfect circle with the radius $\mathrm{a}=30 \mathrm{~km}$ (solid line), (ii) the radius of the heated spot varies from 28.8 to $30.3 \mathrm{~km}$ (dashed line). $W(0)$ is the power in center. Vertical lines mark the limits of the gates, horizontal lines represent the average power inside of each gate and dashed-dotted line shows the estimated level of threshold field.

frequency of $18 \mathrm{MHz}$ and, therefore, the signal was scattered from the density perturbations with transverse scale of $\sim 8.3 \mathrm{~m}$. Significant increase in the scattered power above the noise level is observed in seven gates of the CUTLASS beam 5 (Fig. 1), which was pointed nearly perpendicular to the magnetic field direction. By applying the distribution (8) of the pump power in the heated cone, one can roughly estimate (i) the average pump electric field which acts inside each gate, and (ii) the threshold electric field in the units of the pump electric field. Normalised distribution of the pump power versus distance normalized to the radius of the heated cone is shown in Fig. 2 (solid line). The vertical lines represent the edges of the gates and the horizontal lines represent the average power inside of each gate in the situation when the gates are located symmetrically with respect to the center of the heated cone, which roughly corresponds to the observations shown in Fig. 1. The fact that the scattered signal is observed only in seven gates means that the value of threshold power lies above the average power emitted in the gates located at the distance $1.5<|r / a|<2$ from the centre of the heated spot, but slightly below the power emitted at $|r / a|=1.5$. In this case, some part of the gate with $1.5<|r / a|<2$ can still scatter the HF signal transmitted from the CUTLASS. The heated cone was centred along the direction of the local magnetic field and, therefore, the projection of the heated cone in the horizontal plane at the altitude $230 \mathrm{~km}$ is not a perfect circle with the radius of $30 \mathrm{~km}$ : 

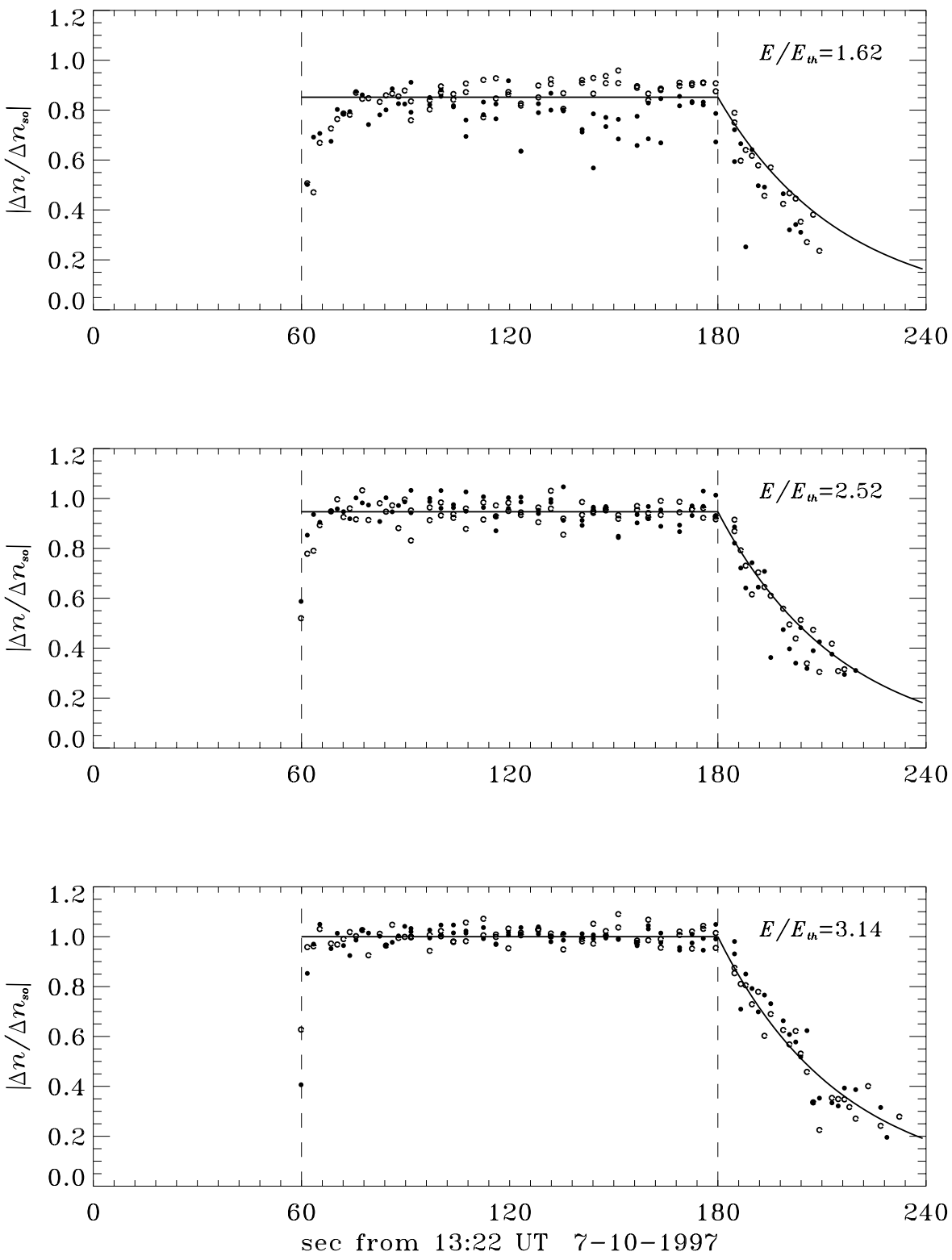

Fig. 3. Temporal variations of the density perturbation normalized to the saturation level observed in the central gates are presented for three ratios of pump electric field to the threshold field. Vertical lines at the times 13:23 UT and 13:25 UT show the moments of switching on and switching off of the $\mathrm{HF}$ heating. The horizontal line marks the mean value of the density perturbations during the heating and represents the saturation level. The solid line after 13:25 UT shows the density decay which is deduced from the fitting of the observational points and follows the exponential law of Eq. (3). the radius of the heated spot varies from 28.8 to $30.3 \mathrm{~km}$. In the situation when the gates are located almost symmetrically with respect to the centre of the heated cone, the gates that are closer to the vertical receive more power than the opposite ones (see the dashed line in Fig. 2) and therefore the HF signal may be scattered from the gate which corresponds to the $r / a$ between 1.5 and 2, but not from the gate between -2 and -1.5 . As a result, the scattered signal is observed in seven gates and the value of the threshold power is estimated to be $\sim 0.09 \mathrm{~W}(0)$ (dashed-dotted line in Fig. 2). Taking into account that the magnitude of the pump electric field is proportional to the square root of the radiated power, the ratio of the average electric field to the threshold value is estimated to be $\sim 3.14 \pm 0.03,2.52 \pm 0.12,1.62 \pm 0.2$ for the gates centered at $\sim 7.5,22.5,37.5 \mathrm{~km}$, respectively, from the center of the heated patch. Since these values are cru- cial for the analysis given below in this section, we introduce the uncertainties in their determination that correspond to the uncertainty of $10 \%$ in the determination of the radius of the heated region.

\subsection{Decay rate}

Let us look at the same data set as shown in Fig. 1, but presented in terms of the density perturbation, which is normalized to the saturation level measured in the central gates. Data shown in each panel (closed and open circles) of Fig. 3 were recorded in two gates located nearly at the same distance from the center of the heated patch and according to the discussion given in the previous section, correspond to the certain ratio of the pump electric field to the threshold field. The vertical lines at the times 13:23 UT and 13:25 UT 

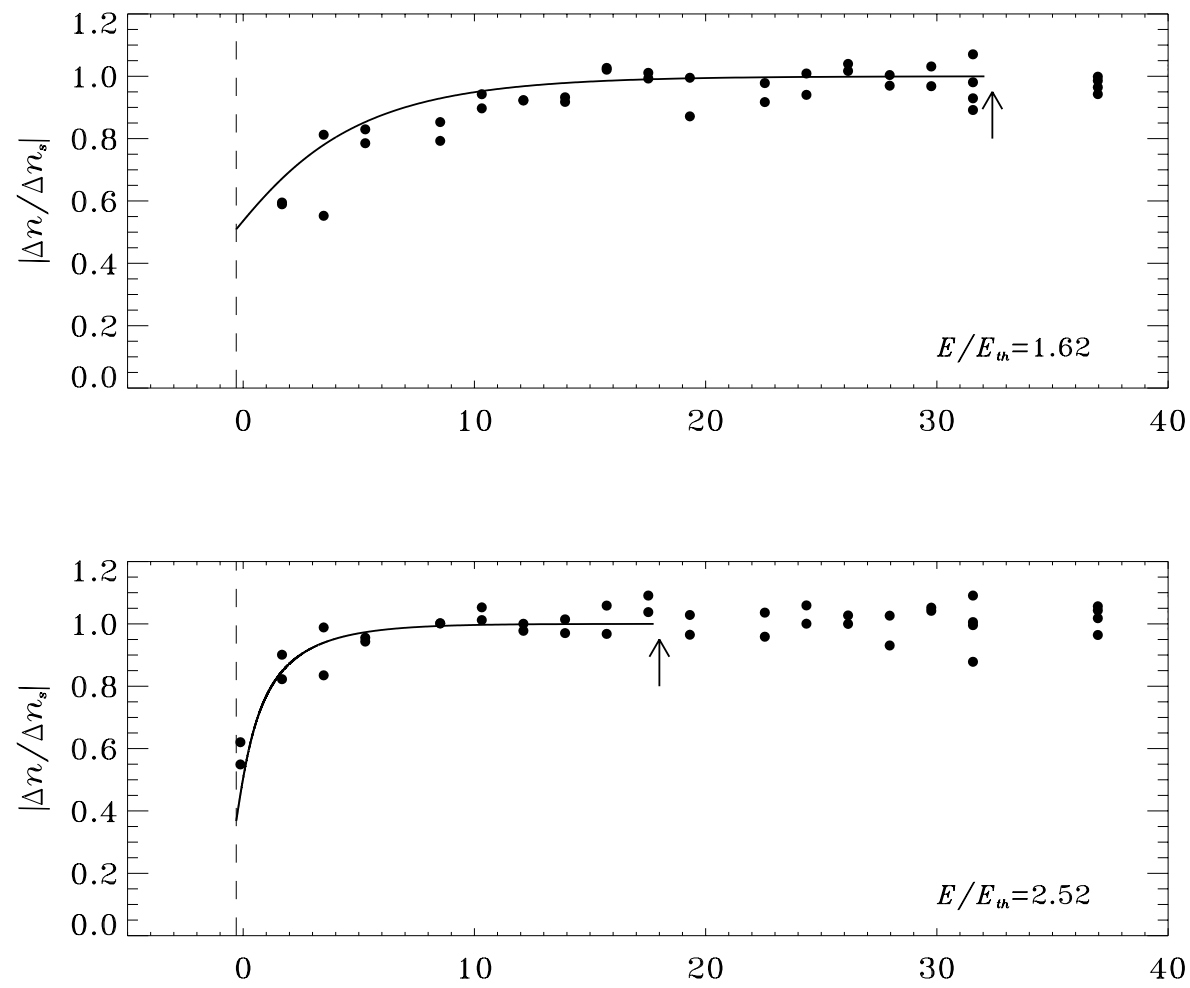

Fig. 4. Density perturbation normalized to the saturation level versus time, as (i) measured by the HF coherent radar (circles) and (ii) estimated from Eq. (2) with the parameters $\sigma \Delta n_{o}^{2} \approx 0.5$ and $D_{\perp} \approx 5 \cdot 10^{-2} \mathrm{~m}^{2} \mathrm{~s}^{-1}$ (solid line), for three ratios of the pump electric field to the threshold level, $E / E_{t h}=3.14$, $2.52,1.62$. Arrows show the estimated time of the stabilisation for each value of pump field. correspond to the moments of the switching on and switching off of the HF heating, respectively. Phenomenologically, the development of the FAI is consistent with the TPI mechanism. When the heater is switched on, the density perturbation begins to grow; it then reaches the saturation level and decays after the heater is switched off. The decay rate does not depend on the pump electric field and is found to follow the exponential law (3). The diffusion coefficient that produces the best fit to these data is estimated to be $D_{\perp} \approx 5 \cdot 10^{-2} \pm 0.8 \cdot 10^{-2} \mathrm{~m}^{2} \mathrm{~s}^{-1}$, which corresponds to an e-folding time of $\sim 36 \pm 5 \mathrm{~s}$. The fact that the diffusion coefficient remains approximately constant during the decay phase demonstrates that electron temperature is not changed significantly during the HF heating whenever it is made with a small power. Uncertainty in the deter- mination of the diffusion coefficient $\Delta D_{\perp}$ might be used to estimate the upper limit of the temperature modification $\Delta T_{e}$, which cannot be resolved from the observations. If the electron-ion collisions are dominant at the upper-hybrid altitude, then the diffusion coefficient varies proportionally to $\sim T_{e}^{-1 / 2}$ (see, for example, Gurevich, 1978) and, therefore, $\Delta T_{e} \approx 2\left(\Delta D_{\perp} / D_{\perp}\right) T_{e} \approx 680 \mathrm{~K}$. In the case when the electron-neutral collisions determine the effective electron collision frequency, the diffusion coefficient changes proportionally to $\sim T_{e}^{2}\left(\sim T_{e}^{3 / 2}\right)$ if the neutral species $\mathrm{N}_{2}(\mathrm{O})$ are the dominant components in the neutral composition of the thermosphere, and then $\Delta T_{e} \approx 0.5\left(\Delta D_{\perp} / D_{\perp}\right) T_{e} \approx 170 \mathrm{~K}$ $\left(\Delta T_{e} \approx 2 / 3\left(\Delta D_{\perp} / D_{\perp}\right) T_{e} \approx 227 \mathrm{~K}\right)$. 


\subsection{Saturation level as a function of pump electric field}

According to the discussion given in Sect. 2, the density perturbation in FAI grows exponentially at the first stage of the heating, but quickly reaches its saturation level, which is proportional to $\sqrt{\sigma^{-1} 1 n\left(E / E_{t h}\right)}$. Under the conditions when the time duration of the heating with constant power (some minutes) is essentially larger than the time of the FAI growth (some tens of seconds), the saturation level of the density perturbations may be reasonably estimated by the averaging of the density perturbations measured during the heating period. Let us first assume that the variable $\sigma$ does not depend on the gate position with respect to the center of the heated patch. Then, the saturation levels calculated from Eq. (7) and normalized by the saturation level, which was observed in the central gates, are $1,0.9 \pm 0.02,0.65 \pm 0.08$ for the neighboring gates with $E / E_{t h}=3.14 \pm 0.03,2.52 \pm 0.12$, $1.62 \pm 0.2$, respectively. The observations presented in Fig. 3 show that these levels are related, such as $1,0.95,0.85$, which is slightly different from the values predicted by the logarithmic law. This means that the term $\sigma$ is not constant, but varies with the position with respect to the center of the heated spot and is larger for the highest pump electric field. This variation is consistent with the density gradient at the altitude of the wave scattering, which is weaker in the center than at the edges of the heated spot (the term $\sigma$ is inversely proportional to the density gradient). This result could be due to the large-scale density defocusing, which, according to the observations of the reflected signal, took place during the considered HF heating.

\subsection{Time of growth as a function of pump electric field}

The time when the density perturbation reaches its stabilization can be estimated numerically by plotting the density perturbation versus time, evaluated from Eq. (2), and assuming that the saturation time corresponds to the moment when the density variation begins to be negligible, i.e. $\partial \Delta n / \partial t<10^{-5}$. In Fig. 4, we present the density perturbation normalized to the saturation level that (i) is measured in three pairs of symmetrical gates and shown by circles, and (ii) is found from Eq. (2) and shown by solid line. The value of the diffusion coefficient is taken from the previous considerations and the estimate of the initial perturbation $\sigma \Delta n_{o}^{2} \approx 0.5$ is chosen to fit the observational points. The characteristic times of the stabilization (shown by the arrows in Fig. 4) are found to be $14.5 \pm 0.5,18 \pm 1$ and $32.4 \pm 7 \mathrm{~s}$ for the ratios with $E / E_{t h}=3.14 \pm 0.03,2.52 \pm 0.12,1.62 \pm 0.2$, respectively, i.e. approximately following the $E^{-1}$ law. The main advantage of the fitting is that it provides the estimates of the initial density perturbations with respect to the saturation level and we conclude that under the HF heating, the amplitude of the density depletion grows in the ratios $\sim 3$, 2.9, 2 under the conditions $E / E_{t h}=3.14,2.52,1.62$, respectively.

It should be mentioned here that although the estimates of the growth time are consistent with the results of the previ- ous publications (see, for example, Frolov et.al, 1997), however, the folding law is estimated to be different. Frolov et al. (1997) considered a large range of the effective pump power with the low limit around the threshold level and found the $E^{-2}$ law. The difference may be due to the strong dependence of the folding law on the amplitude of the pump electric field.

\section{Conclusions}

We have presented a case study of the HF coherent scatter radar observations during the HF heating with small power, which, nevertheless, represents a general behavior of the small-scale FAI developed in the dayside ionosphere at the altitudes around $200 \mathrm{~km}$ and have demonstrated that:

- the slowing of the density growth that is caused by the transverse thermal conductivity leads to the stabilization of the density perturbation in the time which is inversely proportional to the pump electric field;

- the saturation level varies slowly with the pump electric field and follows the logarithmic law;

- HF heating with small power leads to the insignificant modification of the electron temperature (few hundred $\mathrm{K})$ and to the variation of electron density in only a few times.

Overall, our investigations reveal that the simple model of the TPI with three-wave interaction is consistent with the main physical characteristics of the mechanism of instability in the upper-hybrid range.

Acknowledgements. This work was supported by PPARC grant PPA/G/O/1999/00181. We acknowledge the EISCAT staff for operating the facilities.

The Editor in Chief thanks E. Mjolhus and another referee for their help in evaluating this paper.

\section{References}

Bond, G., Robinson, T. R., Eglitis, P., Wright, D. M., Stocker, A. J., Rietveld, M. T., and Jones, T. B.: Spatial observations by the CUTLASS coherent scatter radar of ionospheric modification by high power radio waves, Ann. Geophysicae, 15, 1412-1421, 1997.

Dysthe, K. B., Mjølhus, E., Pécseli, H. L., and Rypdal, K.: A thermal oscillating two-stream instability, Phys. Fluids, 26, 146-157, 1983.

Frolov, V. L., Erukhimov, L. M., Metelev, S. A., and Sergeev, E. N.: Temporal behaviour of artificial small-scale ionospheric irregularities: reveiw of experimental results, J. Atmos. Solar-Terr. Phys., 59, 2317-2333, 1997.

Gurevich, A. V.: Nonlinear phenomena in the ionosphere, (Eds) Roederer, J. G. and Wasson, J. T., Springe, 1978.

Gurevich, A. V., Zybin, K. P., and Lukyanov, A. V.: Stationary striations developed in the ionospheric modification, Phys. Rev. Lett., 75, 2622-2625, 1995. 
Inhester, B.: Thermal modulation of the plasma density in ionospheric heating experiments, J. Atmos. Terr. Phys., 44, 10491059, 1982.

Minkoff, J.: Radio frequency scattering from a heated ionospheric volume, 3, Cross section calculations, Radio Sci., 9, 997-1004, 1974.

Robinson, T. R.: The heating of the high latitude ionosphere by high power radio waves, Physics Reports, 179, 81-209, 1989.

Robinson, T. R., Stocker, A. J., Bond, G., Eglitis, P., Wright, D., and
Jones, T. B.: O and X mode heating effects observed simultaneously with the CUTLASS and EISCAT radars and low power HF diagnostics at Troms $\varnothing$, Ann. Geophysicae, 15, 134-136, 1997.

Robinson, T. R., Stocker, A. J., Bond, G., Eglitis, P., Wright, D., and Jones, T. B.: First CUTLASS-EISCAT heating results, Adv. Space Sci., 21, 663-666, 1998.

Stubbe, P.: Review of ionospheric modification experiments at Troms $\varnothing$, J. Atmos. Terr. Phys., 58, 349-368, 1996. 